\title{
Cerebral embolism secondary to cardiac amyloidosis: A case report and literature review
}

\author{
XU-DONG ZHANG ${ }^{1}$, YING-XIAN LIU ${ }^{2}$, XIAO-WEI YAN ${ }^{2}$, LI-GANG FANG $^{2}$, \\ QUAN FANG $^{2}$, DA-CHUN ZHAO ${ }^{3}$ and YI-NING WANG ${ }^{4}$ \\ Departments of ${ }^{1}$ Respirology, ${ }^{2}$ Cardiology, ${ }^{3}$ Pathology and ${ }^{4}$ Radiology, \\ Peking Union Medical College Hospital, Beijing 100730, P.R. China
}

Received May 19, 2017; Accepted October 12, 2017

DOI: $10.3892 / \mathrm{etm} .2017 .5301$

\begin{abstract}
Cardiac amyloidosis (CA) describes a group of heterogeneous diseases that are characterized by the extracellular fibril deposition of amyloid protein in the myocardium. The abnormal protein is usually derived from light-chain amyloidosis, mutant transthyretin amyloidosis and wild-type transthyretin. Patients with ischemic strokes and amyloidosis have been sporadically reported, however, they are not well summarized. In the present study, a case of cerebral ischemic stroke, secondary to CA was described. This patient presented with dyspnea on exertion, without any evidence of atrial fibrillation. A biopsy revealed deposition of amyloid in the myocardium and Congo Red staining was positive. He suffered from acute infarction of left basal ganglia, resulting from occlusion of the left middle cerebral arterial 6 months prior to admission. However, re-examination of cerebral magnetic resonance imaging in the present hospital revealed an old infarction in the region of the left basal ganglia with a normal appearance of the left middle cerebral artery. Transesophageal echocardiography (TEE) and cardiac magnetic resonance (CMR) both discovered intra-cardiac thrombi, confirming the diagnosis of cardiogenic cerebral embolism. The present study indicates that patients with CA may additionally present with cardiogenic cerebral embolism, and TEE and CMR imaging may help to avoid missing the presence of intra-cardiac thrombi.
\end{abstract}

\section{Introduction}

Immunoglobulin light chain amyloidosis (AL) amyloidosis is a monoclonal plasma cell disorder in which the precursor protein is an immunoglobulin light chain or light chain fragment. It may occur as a primary disease or in association with

Correspondence to: Professor Ying-Xian Liu, Department of Cardiology, Peking Union Medical College Hospital, 1 Shuai Fu Yuan, Dong Cheng, Beijing 100730, P.R. China

E-mail: liuyingxian@pumch.cn

Key words: cardiac amyloidosis, cerebral embolism multiple myeloma or other plasma cell dyscrasias. In primary amyloidosis, there is $2: 1$ preponderance for $\lambda$ over $\kappa$ light chain synthesis (1). AL amyloidosis is the commonest type of amyloidosis, accounting for about $85 \%$ of all newly diagnosed cases. Cardiac amyloidosis (CA) describes a disease in which cardiac extracellular space is expanded by a type of amorphous, fibril protein known as amyloid (2). While 31 subtypes of protein are known to deposit as amyloid, only 11 have been identified involving the heart (3), and AL amyloidosis is the most common subtypes (4). The heart is affected in $>50 \%$ of cases (5), and symptomatic cardiac involvement portends a worse prognosis $(6,7)$. Conversely, involvement limited to the heart constitutes $<5 \%$ of patients with AL amyloidosis. Cardiac contractile function and electrical conduction can be impaired with amyloid infiltration. At a cellular level, amyloid infiltration results in abnormal cellular metabolism, calcium transport and receptor regulation. Cardiac involvement with resultant heart failure or arrhythmia accounts for $>50 \%$ of the mortality in patients with AL amyloidosis $(1,7)$. Histological examination remains the definitive diagnostic modality in CA (8). While not definitive, certain non-invasive imaging and laboratory findings may guide further diagnostic testing and management and assess the severity of the disease for prognostic purposes (9). Appropriate treatment of amyloid disorders requires the correct identification of the subtype of amyloid protein and severity of cardiac involvement, as patients with advanced cardiomyopathy are unsuitable candidates for effective hematological treatment including autologous stem cell transplantation (10).

Thromboembolism also contributes significantly to morbidity and mortality. Intracardiac thrombosis was found in $51 \%$ (11) and 35\% (12) patients with AL-type CA in the Mayo amyloid autopsy study and in a group of patients undergoing follow up echocardiographic imaging respectively. Cardiogenic ischemic stroke is a rare, underappreciated complication of CA. A research for cause of ischemic stroke may contribute to discovery of CA, and therefore benefits for later treatment and prognosis. Herein, we report a patient who suffered from embolic cerebral infarct causing by intra-cardiac thrombus, as a result of $\mathrm{CA}$, and review literatures focusing on documented cases of ischemic stroke secondary to CA, in order to highlight the rare cause of ischemic stroke, emphasize the importance of transesophageal echocardiography (TEE) and 

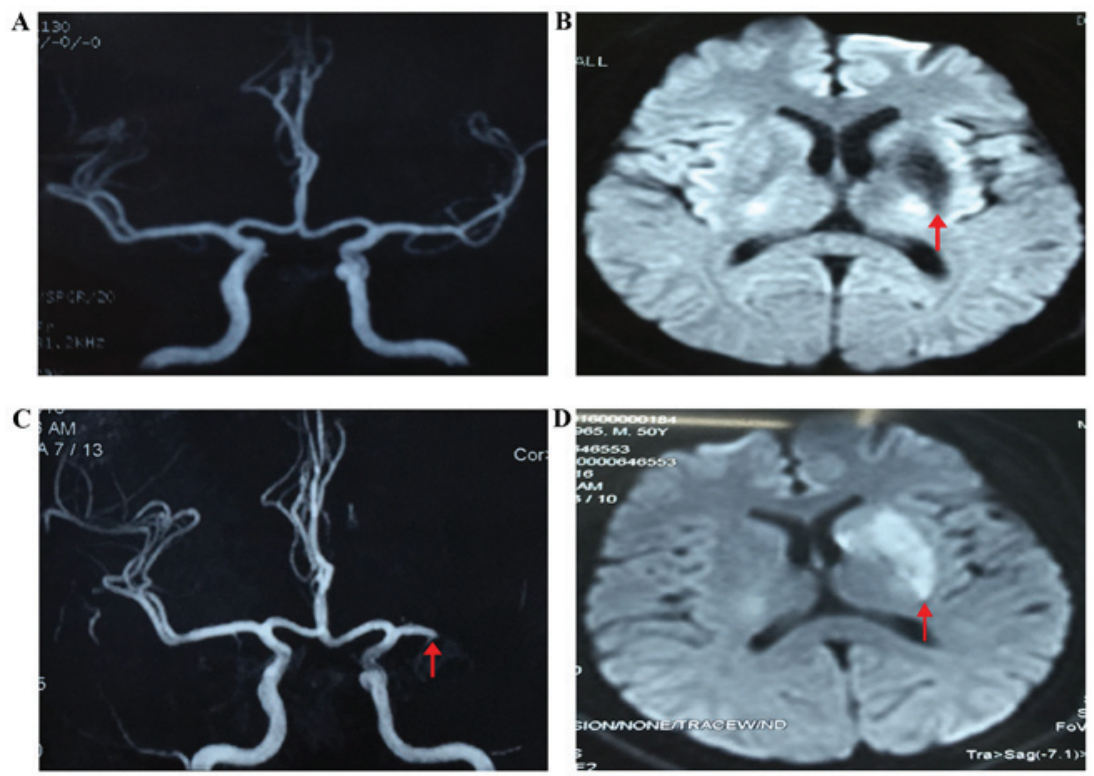

Figure 1. Appearance of MRA and MRI-DWI sequence. (A) Normal appearance of the left MCA; (B) Encephalomalacia loci in the left basal ganglia; (C) Nearly total occlusion of the distal M1 and proximal M2 segment of the left MCA; (D) Cerebral infarcts in the left basal ganglia and corona radiata (red arrow). MRA, magnetic resonance angiography; MRI, magnetic resonance imaging; MCA, middle cerebral artery.

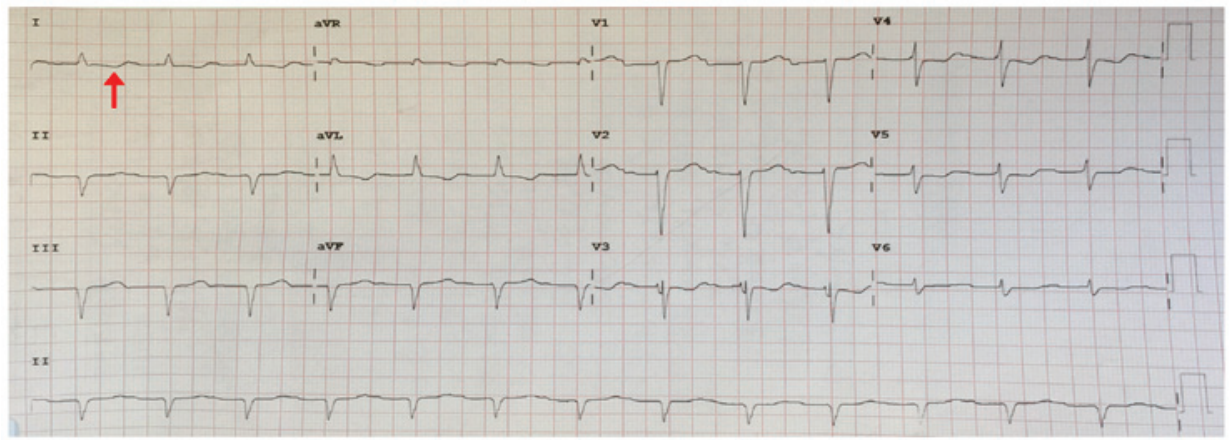

Figure 2. ECG showing one degree atrioventricular block ( $P R$ interval $>0.20 \mathrm{~s}$ ), low voltage in limb leads, left axis deviation, poor R-wave progression in the precordial leads (red arrow).

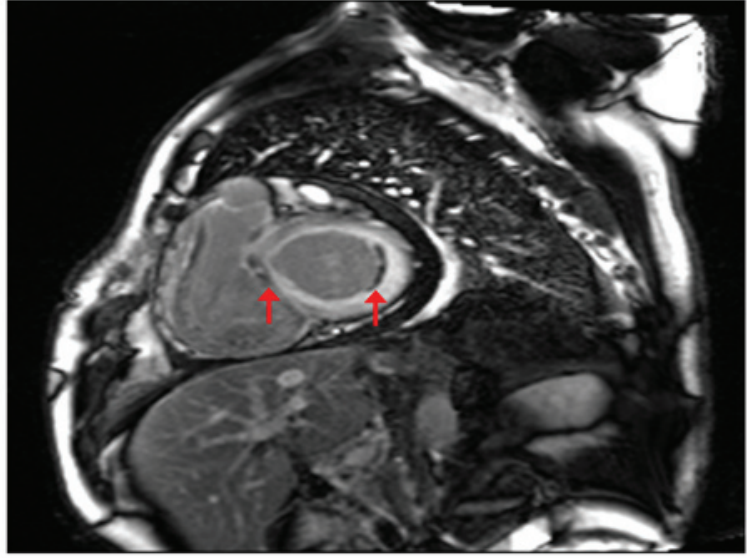

Figure 3. CMR showing subendocardial mural thrombi in the basal segment of left ventricular lateral wall and right ventricular septal wall (red arrow). CMR, cardiac magnetic resonance.

cardiac magnetic resonance (CMR) imaging in discovering intra-cardiac thrombi.

\section{Case report}

A 50-year-old man was admitted to our department presenting with exertional dyspnea for $>1$ year. Physical examination was as follows: BP 100/60 mmHg, HR $90 \mathrm{bpm}$, normal cardiac auscultation with scattered purpura periorbitally and on the neck and abdomen.

Laboratory tests including routine blood culture, hepatic and renal functions, and coagulation test were within normal values. At 6 months before admission, 3 months before admission and at the point of admission, test results were as follows: Uric acid, 266, 364 and $428 \mu \mathrm{mol} / 1$; cardiac troponin I (cTnI), $0.02,0.03$ and $0.05 \mu \mathrm{g} / \mathrm{l} ; \mathrm{N}$-terminal pro-brain natriuretic peptide (NT-proBNP), 2,080, 4,351 and 7,821 pg/ml. Serum immunofixation electrophoresis was normal. Urine immunofixation electrophoresis was negative for free light chain $\kappa(\mathrm{FLC} \kappa)$ and monoclonal protein, but positive for free light chain $\lambda$ (FLC $\lambda$ ). Serum FLC $\kappa$ was $6.93 \mathrm{mg} / \mathrm{dl}$, FLC $140.75 \mathrm{mg} / \mathrm{dl}$, $\kappa / \lambda 0.049 \downarrow$; serum total LC $\kappa 427 \mathrm{mg} / \mathrm{dl} \downarrow$, total LC $\lambda 165 \mathrm{mg} / \mathrm{dl} \downarrow$, $\kappa / \lambda 2.59$. Electromyography showed neurogenic injury of bilateral tibialis anterior muscle. ${ }^{99 \mathrm{~m}} \mathrm{Tc}-\mathrm{MDP}$ bone scan, bone 

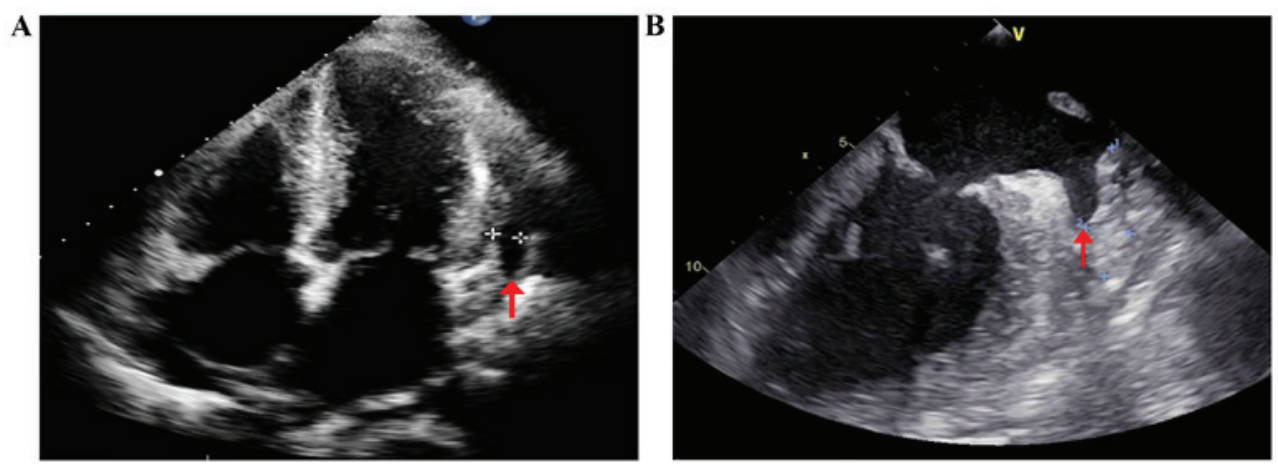

Figure 4. Appearance of echocardiography. (A) TTE showing symmetrical thickening of both ventricular wall with characteristic 'granular sparkling' appearance and a small amount of pericardial effusion (red arrow); (B) TEE showing left auricle appendage thrombus (size 11"37 mm; red arrow). TEE, transesophageal echocardiography.
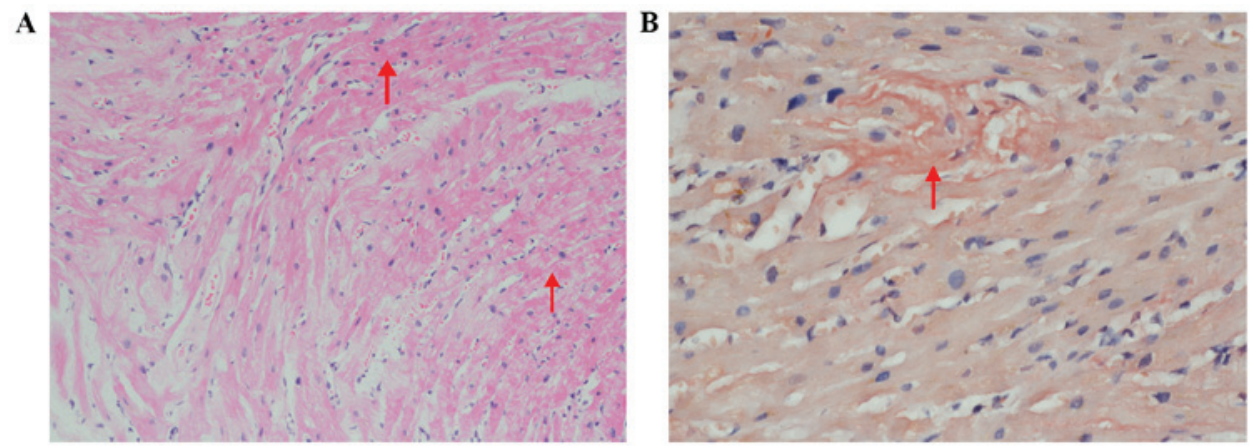

Figure 5. Myocardium biopsy. (A) Hypertrophy in partial cells, nuclear enlargement, fibrous tissue hyperplasiaand degeneration within cardiac muscle fibers, and amyloid deposit in some areas (H\&E, x200 magnification); (B) Special stain showing positive in Congo red stain, supporting the diagnosis of amyloidosis (Congo red stain, x300 magnification) (red arrow).

Table I. Summary of literature reports on CA with ischemic stroke.

\begin{tabular}{lcc}
\hline Author, year & No. of reported patients & Refs. \\
\hline Cho et al, 2005 & 2 & $(13)$ \\
Rice et al, 1981 & 1 & $(14)$ \\
Bøtker et al, 1986 & 1 & $(15)$ \\
Owa et al, 2001 & 1 & $(16)$ \\
Yamano et al, 2008 & 1 & $(17)$ \\
Gillmore et al, 1999 & 1 & $(18)$ \\
Saux et al, 2006 & 1 & $(19)$ \\
Present case, 2016 & 1 & \\
\hline
\end{tabular}

marrow and abdominal fat aspiration biopsies showed no abnormality. Cerebral magnetic resonance imaging (MRI) showed an old infarction in the left basal ganglia, however, the appearance of magnetic resonance angiography (MRA) was normal (Fig. 1A). Electrocardiogram and 24-h Holter monitoring found first degree atrioventricular block (Fig. 2). 6-min walk distance test indicated a reducing exercise capacity (distance walked 330 meters; prediction $=[(7.57 \mathrm{x}$ height in $\mathrm{cm})$ - (5.02 x age) - (1.76 x weight in $\mathrm{kg})$ - 309)]. CMR showed bilateral atrial enlargement, mitral and tricuspid insufficiency, bilateral ventricular diastolic and systolic functional impairment; thickened bilateral atrial and ventricular walls with diffusing delayed enhancement, especially under the endocardium. There was a localized mural thrombus in basal segment of left ventricular lateral wall (Fig. 3). Transthoracic echocardiography (TTE) showed a left ventricular ejection fraction of $46 \%$, symmetrical thickening of both ventricular walls with characteristic 'granular sparkling' appearance; both atrial enlargement and restrictive diastolic dysfunction of the left ventricle (Fig. 4A). TEE showed slow flow and thrombus in the left auricle (Fig. 4B). Amyloid deposits and Congo red staining was positive in myocardial biopsy (Fig. 5).

Based on the above findings, diagnoses of CA (AL-type), restrictive cardiomyopathy and chronic heart failure were made.

Benazepril, spironolactone and metoprolol were prescribed for chronic heart failure; for anticoagulation, with consideration of old cerebral embolism, the risk of recurrent stroke, and the existing thrombus in atrium and left ventricle, low molecular weight heparin was prescribed; bortezomib was chosen as the first line chemotherapy for amyloidosis. Autologous stem cell transplant was under consideration, however, after 6 months, the patient deteriorated and died of decompensate heart failure.

Half a year before admission, the patient visited a local hospital for right extremity aphasia and dyskinesia. Cerebral MRI detected new infarction in the left basal ganglia. MRA showed nearly total occlusion of the distal left middle cerebral artery (MCA) (Fig. 1B). After medical treatment with aspirin and statins, he completely recovered without any neurological 


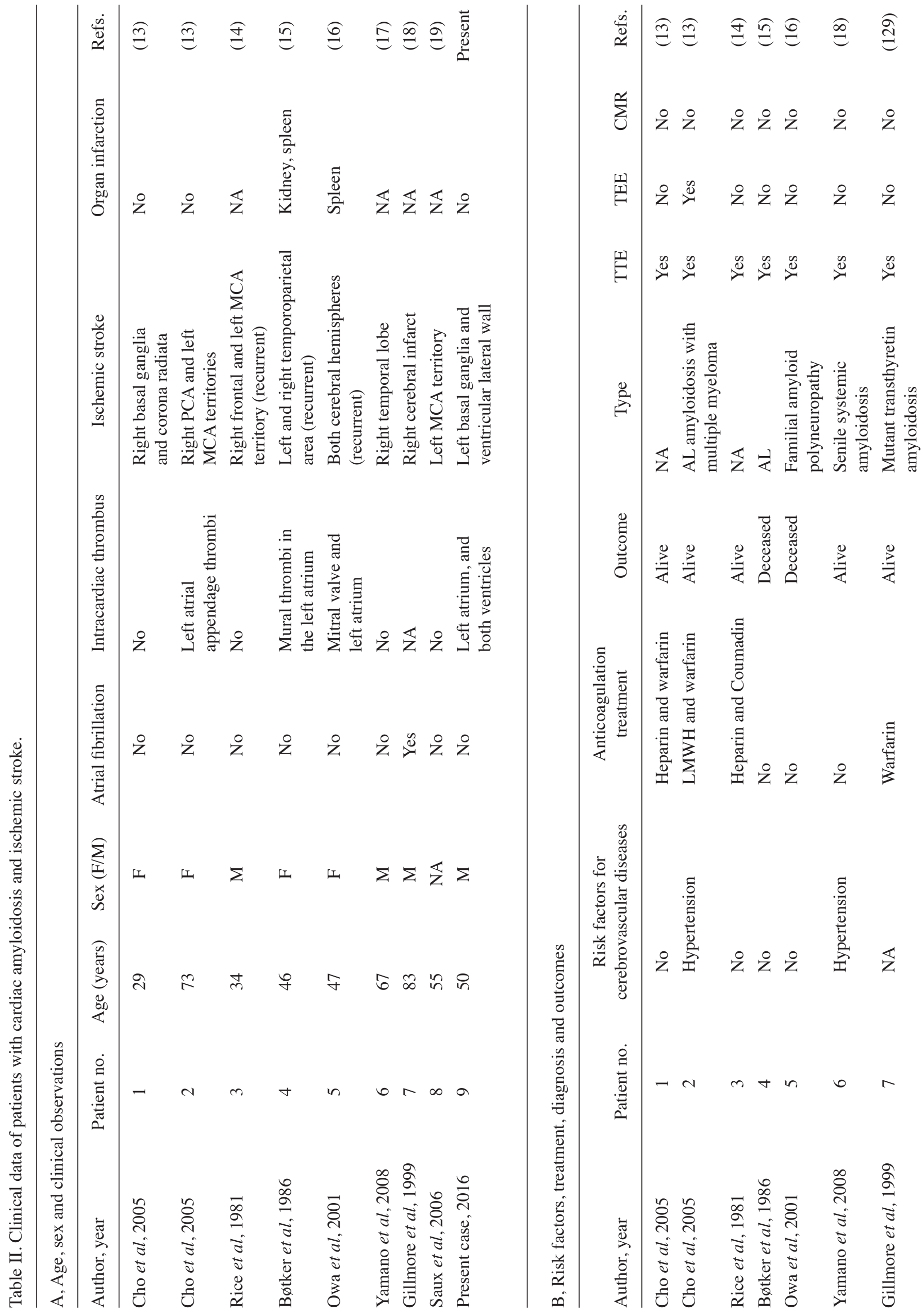


deficits. The patient had no history of hypertension or diabetes. He had never had smoking or drinking habits. Ultrasonic Doppler imaging of carotid and subclavian arteries were normal.

\section{Literature review}

A literature search on Pubmed and Medline database was performed from January 1980 to December 2016 using key words 'cerebral infarct/ischemic stroke/cerebral embolism/cerebral thrombosis', and 'cardiac amyloidosis', and 'heart plus amyloidosis', resulting a total of 35 articles. Case reports were included, and reports without adequate details or full text were excluded. A total of 7 articles with reference information on 9 patients (including our patient in the present study) qualified for the literature review and served as the study population for the purpose of analysis (Table I) (13-19).

In the 9 patients, 4 were male, with a median age of 52.9 years (range, $29-83$ years), and $\sim 80 \%$ of them were diagnosed at $>40$ years of age. AL-type amyloidosis was relatively common, accounting for $57 \%$ of discovered cerebral embolic events, while other types, such as familial amyloidosis and senile systemic amyloidosis were also involved, especially in older patients $(17,18)$.

Symptoms of patients with CA and cerebral embolism were nonspecific, including manifestations of heart failure and ischemic stroke. Recurrent strokes (14-16) happened in one-third of the patients and 2 patients accompanied with infarction of other organs, such as kidney $(15)$ and spleen $(15,16)$. In addition, 4 patients were diagnosed with CA with stroke as the first presentation (15,17-19). AL-type CA with ischemic stroke had worse outcomes, with short-term survival of few months after the stroke events $(15,19)$. By contrast, acquired amyloidosis accounting for $28.6 \%$ of reviewed cases, had better outcomes with longer ages over 65 years, however, it exhibited a high risk of amyloid cardiomyopathy development $(17,18)$.

All 9 patients were negative for intra-cardiac thrombus by TTE. However, 4 of whose (including our case) medical history and electrocardiogram showing no evidence of atrial fibrillation, revealed intra-cardiac thrombus $(13,15,16) .2$ patients were proved by TEE $(13,16), 1$ by autopsy $(16)$, and the other was not elaborated. As to the present case, he was found to have intra-cardiac thrombus by both TEE and CMR.

In addition to the routine treatment of heart failure, 4 patients $(13,16,19)$, including the present case, were prescribed with immunosuppressive therapy, and one patient underwent liver transplantation (16). 5 patients $(13,14,18)$ and the present case received anticoagulation therapy, 4 of whom survived $(13,14,18), 1$ of whom died of heart failure (our case), 1 of whom had recurrent stroke but was still alive (14) at the time of press. Oppositely, 3 of the 4 patients $(15-17,19)$ without anticoagulation therapy died of heart and kidney failure (15), disseminated intravascular coagulation (16) and multiple myeloma (19) respectively. Clinical data of the patients are presented in Table II.

\section{Discussion}

Amyloidosis is a group of diseases that result from the extracellular deposition of amyloid, a fibrillar material derived from various precursor proteins that self-assemble with highly 
ordered abnormal cross $\beta$-sheet conformation (7). Amyloidosis is a multiorgan disease, that results in nonspecific symptoms, including dyspnea, weight loss, edema, proteinuria, bleeding tendency, orthostatic hypotension, and other features of autonomic or peripheral neuropathy. Cardiac involvement is the major determinant of survival. Deposition of amyloid fibrils in the extracellular space causes separation and distortion of the existing tissues and eventually causes irreversible cardiac dysfunction. This may occur in the myocardium, pericardium, small vessels and conduction system (20). When the myocardium is primarily affected, the ventricles may become stiff, leading to restrictive cardiomyopathy. The stiff ventricles may impair ventricular filling during diastole, leading to a clinical presentation of diastolic heart failure, and later systolic dysfunction (20); Angina is common in CA and is thought to be frequently secondary to amyloid deposition in small coronary arteries and infrequently coronary artery disease; Deposition of amyloid can also lead to conduction system dysfunction, with arrhythmias and sudden death $(21,22)$. In our study, clinical features of the patient was accordant with AL amyloidosis with significant heart involvement.

Prognosis of CA is generally poor. For patients with AL amyloidosis, the median survival is 6 months after appearance of congestive heart failure $(13,23)$, however, patients with senile systemic amyloidosis and heart failure live with longer survival of 5 years (24). Cardiac troponins and NT-proBNP are important prognostic indicators in CA, and also allow for monitoring progression of disease or efficacy of therapy (25). Serum uric acid is a novel independent prognostic factor in $\mathrm{AL}$ amyloidosis and the median overall survival was lower in patients with uric acid levels $\geq 8 \mathrm{mg} / \mathrm{dl}$ (26). A combination of uric acid, troponin $\mathrm{T}$ and $\mathrm{N}$-terminal-pro-B-type natriuretic peptide provides a strong predictive model for early mortality (27). The serum immunoglobulin free light chain assay enables quantification of aberrant circulating amyloidogenic fibril protein precursors and serial monitoring of amyloidogenic light chain production during chemotherapy (28). In the present study, cTnI, NT-proBNP and uric acid of our patient (results of other patients were not obtained) were all elevated and progressively increased along with disease exacerbation. Delayed diagnosis leads to prognosis of CA with cerebral embolism even worsen, resulting from the low incidence of cerebral embolism in CA, less cognition of physician and insufficient examination (such as TEE, CMR). The true incidence of ischemic strokes in patients with CA is unknown. One prospective study including 40 patients with systemic amyloidosis and ischemic stroke described 37 of AL, 2 of SSA and 1 of FA; 13 patients presented ischemic stroke as the first manifestation (11 were AL), with average survival of 6.9 months after diagnosis of amyloidosis; $37 \%$ of the 13 patients experienced recurrent ischemic stroke; while $70 \%$ of them had cardioembolic infarctions (29).

Intra-cardiac thrombus in patients of CA are common. Roberts and Waller demonstrated that 14 patients (26\%) were presented with at least one intra-cardiac thrombus in 54 necropsy patients with CA (30). Feng et al discovered 38 patients (33\%) with thrombus through a total of 116 autopsy or transplanted cases of CA (55 of AL and 61 of other type), including 23 of 1 thrombus and 15 of 2-5 thrombi; for a total of 63 thrombi, 36 were in the right atrium, 19 in the left atrium,
4 on the coronary sinus valve, 3 in the right ventricle, and 1 in the left ventricle. The author thought AL type, atrial fibrillation, left ventricular diastolic dysfunction, higher heart rate, right ventricular wall thickness were independently associated with thromboembolism (11). In this study, despite the strong consideration of cerebral embolic stroke, all the reviewed 9 cases were negative of intra-cardiac thrombus by TTE. However, 4 patients was revealed of intra-cardiac thrombus by other methods: 2 patients were proved by TEE $(13,16), 1$ by autopsy (16), while the last one was not described clearly. In addition, our case was also discovered with intra-cardiac thrombus by CMR. Summing up the above, TTE is not as sensitive as enough to display intra-cardiac thrombus, especially special thrombi in the left auricle. So, we suggest that it is necessary to carry out TEE or CMR in CA patients with risk factors of atrial embolism, in order to avoid missing intra-cardiac thrombus or severe cerebral embolic events (31).

Mechanisms of intra-cardiac thrombus in CA includes the following points: First, blood stasis and associated cardiac intracavitary turbulence may contribute to mural thrombosis (15). Second, focal endocardial and subendocardial areas are consistently involved by severe deposition of amyloid, with fibrous thickening and impaired wall motion attributed to organized endocardial thrombi $(14,16)$. Third, arrhythmia is another established culprit of embolic stroke. Atrial fibrillation may due to left atrial dilatation and failure with increasing wall stress, which contributes to thrombosis in left atrium $(13,14)$. Forth, amyloid may infiltrate into the intima of coronary arteries, which may lead to myocardial ischemia, endocardial damage, mural thrombosis and subsequent cerebral embolism (13). Lastly, the plasma hypercoagulability state resulted from nephrotic syndrome, thrombocytosis related to hyposplenism, thrombin-antithrombin pathway impairment or, blood viscosity or procoagulant activity related to circulating monoclonal component may also be a contributor (32).

In fact, due to deficiency of coagulation factors, abnormal fibrin polymerization, hyperfibrinolysis, platelet dysfunction, and vascular damage caused by local amyloid deposition, hemorrhage tendency in patients with amyloidosis is more common than thrombophilia (33). Physical examination also revealed typical cutaneous bleeding, including periorbital, cervical and abdominal purpura in our case. Meanwhile, mural thrombi in left atrium and both ventricles were found, which further even resulted in embolic cerebral infarction.

If intra-cardiac thrombus is detected, anticoagulation therapy may be indicated to prevent systemic circulation embolism; however, anticoagulation therapy may exacerbate the hemorrhagic tendency that is known in amyloidosis due to fragile blood vessel walls secondary to amyloid deposition and the coexisting coagulopathy. Melo et al once reported a case with recurrent cardiac embolic infarcts, developing fatal intracerebral hemorrhage 3 days after intravenous anticoagulation therapy. Anticoagulation therapy and cerebral amyloid angiopathy were demonstrated to trigger cerebral hemorrhage (34). However, in the reviewed 9 patients, 5 patients received anticoagulation therapy, 4 of whom were alive without any visceral hemorrhage. On the contrary, 3 of the 4 patients not prescribed with anticoagulation therapy were deceased, 2 of whom underwent recurrent strokes and other systemic organ infarcts $(15,16)$. These results indicate the probable effect of 
anticoagulation therapy on decreasing the incidence of arterial embolic complications, and benefits to improve prognosis and survival time. Given the unhealed feature and poor prognosis in amyloidosis, more profound researches about occasion to start and terminate, frequency and dose of anticoagulation therapy in CA patients with ischemic stroke are required.

In conclusion, this report presents a case of CA with cardioembolic cerebral stroke although no clues of atrial fibrillation, while subcutaneous bleeding tendency occurs simultaneously. Cardiac embolus is a rare cause of ischemic stroke in adults but could be progressive rapidly, recurrent and fatal. Summarizing the key aspects of this rare disease based on a review of available literatures, we emphasize the importance of making a thorough search for cardiac problems in all stroke patients with low risk of cerebrovascular diseases, and the crucial role of TEE and CMR in detecting the intra-cardiac thrombus of CA patients.

\section{References}

1. Shah KB, Inoue Y and Mehra MR: Amyloidosis and the heart: A comprehensive review. Arch Intern Med 166: 1805-1813, 2006.

2. Falk RH, Alexander KM, Liao R and Dorbala S: AL (light-chain) cardiac amyloidosis: A review of diagnosis and therapy. J Am Coll Cardiol 68: 1323-1341, 2016.

3. Maleszewski JJ: Cardiac amyloidosis: Pathology, nomenclature, and typing. Cardiovasc Pathol 24: 343-350, 2015.

4. Rosenbaum AN and Edwards BS: Current indications, strategies, and outcomes with cardiac transplantation for cardiac amyloidosis and sarcoidosis. Curr Opin Organ Transplant 20: 584-592, 2015.

5. Merlini G: CyBorD: Stellar response rates in AL amyloidosis Blood 119: 4343-4345, 2012.

6. Desport E, Bridoux F, Sirac C, Delbes S, Bender S, Fernandez B, Quellard N, Lacombe C, Goujon JM, Lavergne D, et al: Al amyloidosis. Orphanet J Rare Dis 7: 54, 2012.

7. Wechalekar AD, Gillmore JD and Hawkins PN: Systemic amyloidosis. Lancet 387: 2641-2654, 2016.

8. Wechalekar AD, Gillmore JD and Hawkins PN: Cardiac amyloidosis: An approach to diagnosis and management. Expert Rev Cardiovasc Ther 8: 1007-1013, 2010.

9. Selvanayagam JB, Hawkins PN, Paul B, Myerson SG and Neubauer S: Evaluation and management of the cardiac amyloidosis. J Am Coll Cardiol 50: 2101-2110, 2007.

10. Karafiatova L and Pika T: Amyloid cardiomyopathy. Biomed Pap Med Fac Univ Palacky Olomouc Czech Repub 161: 117-127, 2017.

11. Feng D, Edwards WD, Oh JK, Chandrasekaran K, Grogan M Martinez MW, Syed IS, Hughes DA, Lust JA, Jaffe AS, et al: Intracardiac thrombosis and embolism in patients with cardiac amyloidosis. Circulation 116: 2420-2426, 2007.

12. Feng D, Syed IS, Martinez M, Oh JK, Jaffe AS, Grogan M, Edwards WD, Gertz MA and Klarich KW: Intracardiac thrombosis and anticoagulation therapy in cardiac amyloidosis. Circulation 119: 2490-2497, 2009.

13. Cho KH, Cho YM and Kim JS: Embolic infarction associated with cardiac amyloidosis. J Clin Neurol 1: 92-96, 2005.

14. Rice GP, Ebers GC, Newland F and Wysocki GP: Recurrent cerebral embolism in cardiac amyloidosis. Neurology 31: 904-907, 1981.

15. Bøtker HE and Rasmussen OB: Recurrent cerebral embolism in cardiac amyloidosis. Int J Cardiol 13: 81-83, 1986.

16. Owa M, Takei Y, Hashikura Y, Kawasaki S, Koyama M and Ikeda S: Recurrent cerebral embolism in a familial amyloid polyneuropathy patient who received partial liver transplantation from a living donor. Intern Med 40: 259-264, 2001.
17. Yamano M, Azuma A, Yazaki M, Ikeda S, Sawada T and Matsubara H: Early cardiac involvement in senile systemic amyloidosis: A case report. Amyloid 15: 54-59, 2008.

18. Gillmore JD, Booth DR, Pepys MB and Hawkins PN: Hereditary cardiac amyloidosis associated with the transthyretin Ile122 mutation in a white man. Heart 82: e2, 1999.

19. Saux A, Heroum C, Sportouch C, De Graeve F, Lequellec A and Pagès M: Amyloid cardiomyopathy: A rare cause of cerebral embolism. Rev Neurol (Paris) 162: 1128-1130, 2006 (In French).

20. Yusuf SW, Solhpour A, Banchs J, Lopez-Mattei JC, Durand JB, Iliescu C, Hassan SA and Qazilbash MH: Cardiac amyloidosis. Expert Rev Cardiovasc Ther 12: 265-277, 2014.

21. Ritts AJ, Cornell RF, Swiger K, Singh J, Goodman S and Lenihan DJ: Current concepts of cardiac amyloidosis: Diagnosis, clinical management, and the need for collaboration. Heart Fail Clin 13: 409-416, 2017.

22. Mankad AK, Sesay I and Shah KB: Light-chain cardiac amyloidosis. Curr Probl Cancer 41: 144-156, 2017.

23. Rahman JE, Helou EF, Gelzer-Bell R, Thompson RE, Kuo C, Rodriguez ER, Hare JM, Baughman KL and Kasper EK: Noninvasive diagnosis of biopsy-proven cardiac amyloidosis. J Am Coll Cardiol 43: 410-415, 2004.

24. Cacoub P, Axler O, De Zuttere D, Hausfater P, Amoura Z, Walter S, Wechsler B, Godeau P and Piette JC: Amyloidosis and cardiac involvement. Ann Med Interne (Paris) 151: 611-617, 2000.

25. Palladini G, Barassi A, Klersy C, Pacciolla R, Milani P, Sarais G, Perlini S, Albertini R, Russo P, Foli A, et al: The combination of high-sensitivity cardiac troponin $\mathrm{T}$ (hs-cTnT) at presentation and changes in N-terminal natriuretic peptide type B (NT-proBNP) after chemotherapy best predicts survival in AL amyloidosis. Blood 116: 3426-3430, 2010.

26. Kumar S, Dispenzieri A, Lacy MQ, Hayman SR, Leung N, Zeldenrust SR, Buadi FK, Kyle RA, Rajkumar SV and Gertz MA: Serum uric acid: Novel prognostic factor in primary systemic amyloidosis. Mayo Clin Proc 83: 297-303, 2008.

27. Kumar SK, Gertz MA, Lacy MQ, Dingli D, Hayman SR, Buadi FK, Short-Detweiler K, Zeldenrust SR, Leung N, Greipp PR, et al: Recent improvements in survival in primary systemic amyloidosis and the importance of an early mortality risk score. Mayo Clin Proc 86: 12-18, 2011.

28. Dispenzieri A, Lacy MQ, Katzmann JA, Rajkumar SV, Abraham RS, Hayman SR, Kumar SK, Clark R, Kyle RA, Litzow MR, et al: Absolute values of immunoglobulin free light chains are prognostic in patients with primary systemic amyloidosis undergoing peripheral blood stem cell transplantation. Blood 107: 3378-3383, 2006.

29. Zubkov AY, Rabinstein AA, Dispenzieri A and Wijdicks EF: Primary systemic amyloidosis with ischemic stroke as a presenting complication. Neurology 69: 1136-1141, 2007.

30. Roberts WC and Waller BF: Cardiac amyloidosis causing cardiac dysfunction: Analysis of 54 necropsy patients. Am J Cardiol 52: 137-146, 1983.

31. Santarone M, Corrado G, Tagliagambe LM, Manzillo GF, Tadeo G, Spata M and Longhi M: Atrial thrombosis in cardiac amyloidosis: Diagnostic contribution of transesophageal echocardiography. J Am Soc Echocardiogr 12: 533-536, 1999.

32. Hausfater P, Costedoat-Chalumeau N, Amoura Z, Cacoub P, Papo T, Grateau G, Leblond V, Godeau P and Piette JC: AL cardiac amyloidosis and arterial thromboembolic events. Scand J Rheumatol 34: 315-319, 2005

33. Sucker C, Hetzel GR, Grabensee B, Stockschlaeder M and Scharf RE: Amyloidosis and bleeding: Pathophysiology, diagnosis, and therapy. Am J Kidney Dis 47: 947-955, 2006.

34. Melo TP, Bogousslavsky J, Regli F and Janzer R: Fatal hemorrhage during anticoagulation of cardioembolic infarction: Role of cerebral amyloid angiopathy. Eur Neurol 33: 9-12, 1993. 